\title{
Stepwise Evaluation of Unexplained Syncope in a Large Ambulatory Population
}

\author{
JUAN F. IGLESIAS, M.D., DENIS GRAF, M.D., ANDREI FORCLAZ, M.D., \\ JUERG SCHLAEPFER, M.D., MARTIN FROMER, M.D., and ETIENNE PRUVOT, M.D. \\ From the Unité des Troubles du Rythme, Service de Cardiologie, Lausanne University Hospital, \\ Lausanne, Switzerland
}

Background: Up to $60 \%$ of syncopal episodes remain unexplained. We report the results of a standardized, stepwise evaluation of patients referred to an ambulatory clinic for unexplained syncope.

Methods and Results: We studied 939 consecutive patients referred for unexplained syncope, who underwent a standardized evaluation, including history, physical examination, electrocardiogram, headup tilt testing (HUTT), carotid sinus massage (CSM) and hyperventilation testing (HYV). Echocardiogram and stress test were performed when underlying heart disease was initially suspected. Electrophysiological study (EPS) and implantable loop recorder (ILR) were used only in patients with underlying structural heart disease or major unexplained syncope. We identified a cause of syncope in $66 \%$ of patients, including $27 \%$ vasovagal, $14 \%$ psychogenic, $6 \%$ arrhythmias, and $6 \%$ hypotension. Noninvasive testing identified $92 \%$ and invasive testing an additional $8 \%$ of the causes. HUTT yielded $38 \%$, CSM $28 \%$, HYV $49 \%$, EPS $22 \%$, and ILR $56 \%$ of diagnoses. On average, patients with arrhythmic causes were older, had a lower functional capacity, longer P-wave duration, and presented with fewer prodromes than patients with vasovagal or psychogenic syncope.

Conclusions: A standardized stepwise evaluation emphasizing noninvasive tests yielded 2/3 of causes in patients referred to an ambulatory clinic for unexplained syncope. Neurally mediated and psychogenic mechanisms were behind $>50 \%$ of episodes, while cardiac arrhythmias were uncommon. Sudden syncope, particularly in older patients with functional limitations or a prolonged P-wave, suggests an arrhythmic cause. (PACE 2009; 32:S202-S206)

\section{unexplained syncope, standardized stepwise evaluation, diagnostic testing, psychogenic pseudo-syncope, $P$-wave duration}

\section{Introduction}

Syncope is a common disorder, which remains unexplained in up to $60 \%$ of patients referred to an emergency department. ${ }^{1,2}$ The investigation of unexplained syncope remains challenging. Some investigators have recently shown the superior performance of simple investigations in patients with syncope evaluated in emergency departments. ${ }^{1-3}$ However, little is known of the diagnostic performance of dedicated functional tests. We have shown, in a recent study of the first 317 patients referred to our ambulatory syncope clinic, that the investigation of unexplained syncope, using a standardized stepwise patient evaluation, yielded a diagnosis in $81 \%$ of patients, and that compared with patients whose syncope was neurally mediated (reflex). Those whose cause of syncope was an arrhythmia were older, had fewer prodromes, and had a longer P-wave duration. ${ }^{4}$ This study, which includes the first 900 ambulatory patients referred to our syncope clinic, (1) ex-

No conflict of interest to disclose

Address for reprints: Etienne Pruvot, M.D., Service de Cardiologie, Lausanne University Hospital, Rue du Bugnon 46, 1011 Lausanne, Switzerland. Fax: +41-21-314-51-96; e-mail: etienne.pruvot@chuv.ch amined the diagnostic performance of a standardized stepwise investigation of unexplained syncope, and (2) compared the clinical characteristics of patients with arrhythmic versus reflex causes of syncope.

\section{Patient Population and Methods}

This prospective single center study was conducted in the ambulatory syncope clinic of Lausanne University Hospital, in Switzerland. Patients were referred for investigation of unexplained syncope or presyncope. The former was defined as a transient and self-limited loss of consciousness with inability to maintain postural tone and the latter as a near syncopal event. ${ }^{5}$ Patients with disease manifestations consistent with other disorders, such as seizure, vertigo, or coma, were excluded. The final population comprised 939 consecutive ambulatory patients referred to our syncope clinic for evaluation and management of unexplained syncope between December 1999 and October 2007.

Figure 1 illustrates our standardized stepwise patient evaluation. They underwent a baseline head-up tilt test (HUTT), followed by carotid sinus massage (CSM) in the supine and upright positions, and HUTT with intravenous adenosine and

(C)2009, The Authors. Journal compilation (C)2009 Wiley Periodicals, Inc. 


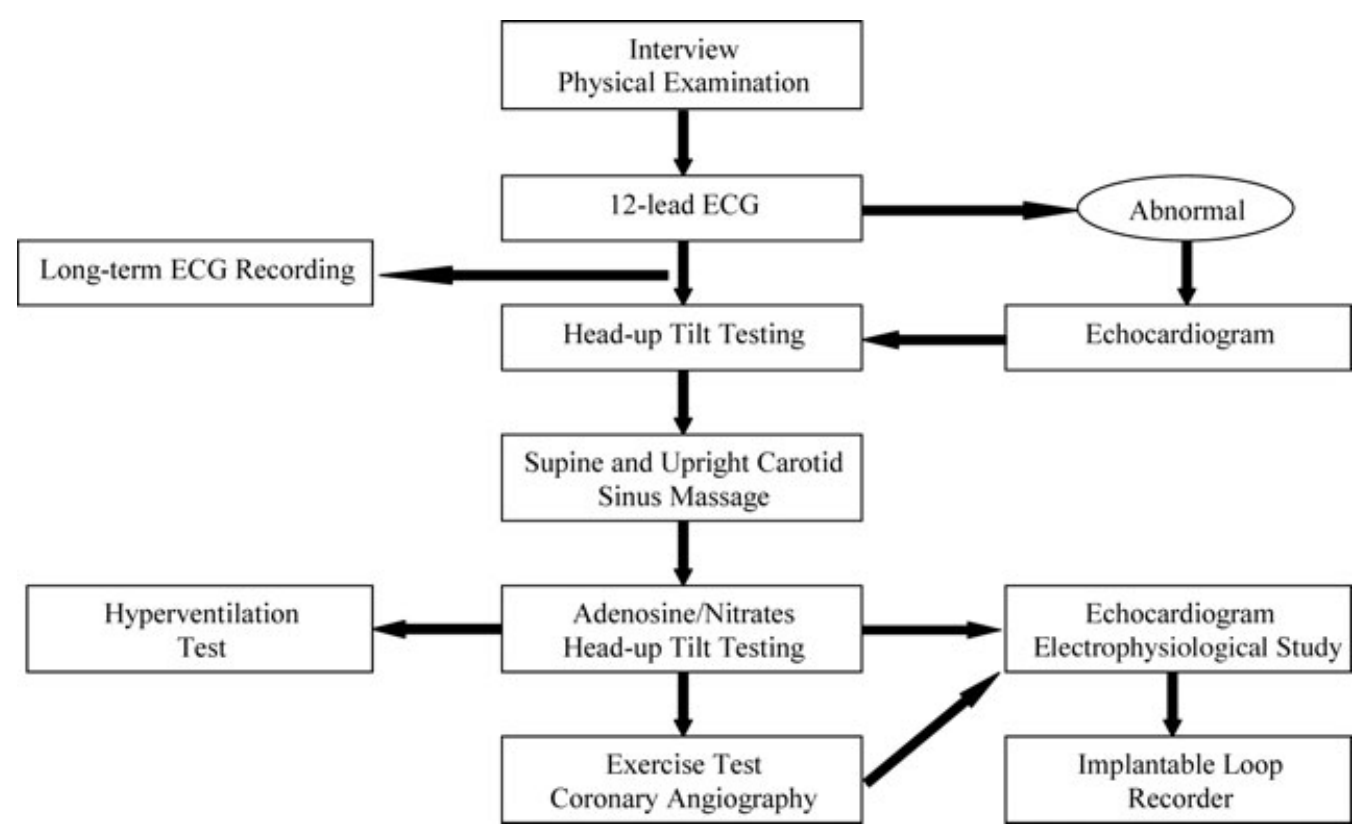

Figure 1. Standardized stepwise patient evaluation.

sublingual nitrates challenges. A hyperventilation test (HYV) was performed only in patients presenting with phobic, anxious, or depressive manifestations. Structural heart disease was ruled out on the basis of patient's history, physical examination and 12-lead electrocardiogram (ECG). Exercise testing and echocardiogram were performed when an underlying cardiac disorder was suspected. An electrophysiological study (EPS) was performed in patients presenting with an underlying cardiac disease or in patients whose initial noninvasive evaluation was negative, and who required further investigations. An implantable loop recorder (ILR; Reveal ${ }^{\circledR}$ Medtronic Inc., Minneapolis, MN, USA) was proposed to patients who had undergone a negative evaluation and suffered syncope-related complications. Importantly, a positive test was classified as diagnostic when the test-induced symptoms reproduced the clinical presentation; otherwise, the test was considered abnormal, though nondiagnostic.

The initial interview focused systematically on the presence and duration of prodromes and recovery manifestations, including nausea, diaphoresis, blurred vision, paresthesia, vertigo, palpitation, anxiety, tongue biting, and confusion. Syncope-related trauma was classified as major, when associated with a bone fracture, head or internal organ injury, or a car accident; otherwise, it was classified as minor. The P-wave duration was measured before any investigation to avoid a methodological bias, by averaging three consecutive cycles from leads II and $\mathrm{AV}_{\mathrm{L}}$.
The causes of syncope were classified according to the guidelines of the European Society of Cardiology. ${ }^{5}$ The ultimate cause of syncope was assigned by one of the investigators (E.P.) at the end of the stepwise evaluation. For statistical analyses, the ultimate cause of syncope was classified as (1) arrhythmic, including bradyarrhythmias due to atrioventricular block or sick sinus syndrome, supra- or ventricular tachyarrhythmias, and cardioinhibitory carotid sinus syndrome (CSS); (2) Vasovagal or psychogenic, including tiltinduced syncope and psychogenic pseudosyncope; (3) Hypotensive, including orthostatic hypotension and vasodepressive CSS; (4) Miscellaneous, including situational syncope and neurological disorders; and (5) unexplained, when none of the above was applicable.

\section{Statistical Analysis}

Continuous variables were compared using Student's $t$-test. Categorical variables were compared using the $\chi^{2}$ test. A P value $<0.01$ was considered statistically significant.

\section{Results}

\section{Clinical Characteristics and Causes of Syncope}

During the study period, 939 consecutive outpatients were enrolled. Table I shows the baseline clinical characteristics of the study population. Their mean age was 52 years, and mean number of syncopal episodes was 10 , with $13 \%$ of patients referred for presyncope only. Syncope was the cause 


\section{Table I.}

Baseline Characteristics of the Study Population

\begin{tabular}{lc}
\hline Age (y) & $52 \pm 21$ \\
Women & $461(49)$ \\
Hypertension & $261(28)$ \\
Hypercholesterolemia & $257(27)$ \\
Diabetes mellitus & $71(8)$ \\
Active smoker & $216(23)$ \\
Ischemic heart disease & $77(8)$ \\
Syncope frequency & $10 \pm 35$ \\
Presyncope only & $120(13)$ \\
Years elapsed since first episode & $5 \pm 8$ \\
Minor trauma & $251(27)$ \\
Major trauma & $105(11)$ \\
Hospitalization & $497(53)$ \\
Car accident & $60(6)$ \\
Driving license withdrawal & $33(3.5)$
\end{tabular}

Values are means \pm standard deviation or numbers (\%) of observations.

of hospitalization in $53 \%$, car accident in $6 \%$, and driving license withdrawal in $3.5 \%$ of the patients. Table II shows the final causes of syncope, diagnosed after the standardized stepwise evaluation. Overall, a final diagnosis was established in $66 \%$, while syncope remained unexplained in $34 \%$. Importantly, arrhythmic causes were uncommon $(6 \%)$, while neurally mediated syncope was diagnosed in $42 \%$, including tilt-induced syncope in

Table II.

Final Causes of Syncope

\begin{tabular}{lc}
\hline & $\mathbf{n}(\%)$ \\
\hline Neurally mediated & $396(42)$ \\
Vasovagal (tilt-induced) & $258(27)$ \\
Situational & $15(2)$ \\
Vasodilatative carotid sinus syndrome & $27(3)$ \\
Cardio-inhibitory carotid sinus syndrome & $96(10)$ \\
Psychogenic pseudosyncope & $129(14)$ \\
Orthostatic hypotension & $31(3)$ \\
Cardiac arrhythmias & $52(6)$ \\
Tachyarrhythmias & $35(4)$ \\
Supraventricular tachycardia & $19(2)$ \\
Ventricular tachycardia & $16(2)$ \\
Bradyarrhythmias & $17(2)$ \\
Sick sinus syndrome & $9(1)$ \\
AV block & $8(1)$ \\
Miscellaneous & $12(1)$ \\
Unexplained & $319(34)$ \\
\hline
\end{tabular}

$27 \%$. Cardioinhibitory CSS was diagnosed in 96 patients $(10 \%)$ and psychogenic pseudo-syncope in $131(14 \%)$, with spontaneous hysterical conversion or panic attacks in $22 \%$ and triggered by HYV in $78 \%$ of these 131 patients. The distribution of final diagnoses according to age $<70$ years $(\mathrm{n}=241)$ versus $\geq 70$ years $(\mathrm{n}=78)$ and men $(\mathrm{n}=$ $168)$ versus women $(\mathrm{n}=151)$ was analyzed. Syncope remained unexplained in $1 / 3$ of all cases, independent of age $(34 \%$ vs $33 \%$, respectively) and gender (35\% vs $33 \%$, respectively). In patients $<70$ years of age, vasovagal and psychogenic causes combined were significantly more prevalent (49\%) and arrhythmic and hypotensive causes less prevalent ( $10 \%$ and $4 \%$, respectively) than in patients $\geq 70$ years of age $(17 \%, 34 \%$, and $13 \%$, respectively, $\mathrm{P}<0.01$ ).

\section{Diagnostic Yield of Individual Tests}

Table III shows the diagnostic yield of individual tests. Since the standardized evaluation was applied sequentially, not all tests were systematically performed. Among noninvasive tests, HYV yielded the best diagnostic performance (49\%) followed by HUTT (38\%), CSM (28\%), and longterm ECG recordings $(28 \%)$. HYV and long-term ECG recordings were used selectively, in patients with psychiatric manifestations and $\geq 1$ episode of syncope/week. Among invasive tests, the diagnostic performance of EPS was poor $(22 \%)$ despite a strict patient selection. In contrast, ILR yielded a diagnosis in 30 of 54 patients (56\%), who presented with syncope-related complications and a negative noninvasive diagnostic evaluation. An arrhythmic cause was confirmed in $20 \%$ of patients, including sick sinus syndrome in $11 \%$, and supraventricular tachyarrhythmias in $9 \%$. A hypotensive cause was suspected in $17 \%$ on the basis of the ILR recorded heart rate

\begin{tabular}{lc}
\hline \multicolumn{2}{c}{ Table III. } \\
\multicolumn{2}{c}{ Diagnostic Yield of Individual Tests } \\
\hline Electrocardiogram & $10 / 939(1)$ \\
Orthostatic blood pressure test & $37 / 870(4)$ \\
Head-up tilt test & $317 / 844(38)$ \\
Carotid sinus massage & $200 / 714(28)$ \\
Hyperventilation test & $140 / 284(49)$ \\
Long-term electrocardiogram & $16 / 58(28)$ \\
Echocardiogram & $10 / 143(7)$ \\
Exercise test & $8 / 70(12)$ \\
Electrophysiological study & $35 / 156(22)$ \\
Implantable loop-recorder & $30 / 54(56)$ \\
\end{tabular}

Values are numbers of diagnostic tests/number of tests performed (\% yield). 
Table IV.

Baseline Clinical Characteristics and Distribution of Statistically* Significant Prodromes According to Causes of Syncope

\begin{tabular}{lcccccc}
\hline & $\begin{array}{c}\text { Arrhythmia } \\
\mathbf{n = 1 4 8}\end{array}$ & $\begin{array}{c}\text { VV/Psy } \\
\mathbf{n = 3 8 7}\end{array}$ & $\begin{array}{c}\text { Hypotension } \\
\mathbf{n = 5 8}\end{array}$ & $\begin{array}{c}\text { Miscellaneous } \\
\mathbf{n = 2 7}\end{array}$ & $\begin{array}{c}\text { Unexplained } \\
\mathbf{n = 3 1 9}\end{array}$ & $\begin{array}{c}\text { Total } \\
\mathbf{n}=\mathbf{9 3 9}\end{array}$ \\
\hline Age (year) & $67 \pm 11$ & $43 \pm 16$ & $64 \pm 15$ & $55 \pm 14$ & $53 \pm 21$ & $52 \pm 21$ \\
Women & $58(39)$ & $224(58)$ & $20(34)$ & $8(30)$ & $151(47)$ & $461(49)$ \\
Hypertension & $65(44)$ & $56(15)$ & $26(44)$ & $9(33)$ & $105(33)$ & $261(28)$ \\
Ischemic heart disease & $29(20)$ & $13(3)$ & $10(17)$ & $1(4)$ & $24(8)$ & $77(8)$ \\
NYHA class $\geq$ II & $44(30)$ & $14(4)$ & $19(32)$ & $6(22)$ & $31(10)$ & $114(12)$ \\
P-wave duration (ms) & $112 \pm 16$ & $98 \pm 12$ & $113 \pm 16$ & $108 \pm 12$ & $103 \pm 16$ & $103 \pm 15$ \\
QRS duration (ms) & $97 \pm 15$ & $88 \pm 11$ & $95 \pm 16$ & $92 \pm 11$ & $92 \pm 15$ & $91 \pm 14$ \\
No prodrome & $69(47)$ & $25(6)$ & $15(25)$ & $5(19)$ & $135(42)$ & $249(27)$ \\
Nausea/vomiting & $19(13)$ & $129(33)$ & $6(10)$ & $3(11)$ & $36(11)$ & $193(21)$ \\
Diaphoresis & $42(28)$ & $316(82)$ & $19(33)$ & $11(41)$ & $104(33)$ & $492(52)$ \\
Blurred vision & $26(18)$ & $191(49)$ & $27(46)$ & $8(30)$ & $82(25)$ & $334(36)$ \\
Paresthesia & $2(1)$ & $85(22)$ & $2(3)$ & $1(4)$ & $15(5)$ & $105(11)$ \\
Vertigo/dizziness & $54(36)$ & $235(61)$ & $28(47)$ & $17(63)$ & $92(29)$ & $426(45)$ \\
Palpitations & $9(6)$ & $82(21)$ & $4(7)$ & $4(15)$ & $29(9)$ & $128(14)$ \\
\hline
\end{tabular}

Mean \pm standard deviation, unmarked data are $n(\%)$.

${ }^{*} P<0.01$ in the comparison of Rhythm, VV/Psy and other three categories pooled.

$\mathrm{VV} / \mathrm{Psy}=$ pooled vasovagal syncope and psychogenic pseudo-syncope; NYHA = New York Heart Association.

during clinical events and confirmed by selected tests. The remaining causes of syncope included vasovagal (9\%), epilepsy (6\%), situational $(2 \%)$, and psychogenic $(2 \%)$. It is noteworthy that the three patients diagnosed with epilepsy had a negative neurological evaluation, including electroencephalogram before ILR implantation. Importantly, $92 \%$ of the final causes of syncope were identified using noninvasive tests, while invasive test yielded diagnoses in an additional $8 \%$ of patients.

\section{Clinical Characteristics and Distribution of Prodromes According to the Final Diagnosis}

Table IV shows the baseline clinical characteristics and distribution of statistically significant prodromes according to causes of syncope. Compared with vasovagal syncope and psychogenic pseudo-syncope, patients with arrhythmic causes were older, had a lower functional capacity, a higher prevalence of hypertension and ischemic heart disease, a longer P-wave but similar QRS duration, and suffered more often from sudden syncope. Prodromes, such as nausea, diaphoresis, paresthesia, palpitations, and anxiety, were significantly more common in patients with vasovagal syncope and psychogenic pseudo-syncope than in patients with arrhythmic causes or other categories.

\section{Discussion \\ Distribution of Causes of Syncope}

The application of a standardized stepwise diagnostic evaluation identified a cause of syncope in $66 \%$ of patients. The distribution of final diagnoses is concordant with previous reports. ${ }^{6}$ Vasovagal (HUTT-induced) syncope was the most prevalent cause, while cardiac arrhythmias were uncommon. The higher prevalence of vasovagal syncope in younger and arrhythmic causes in older patients is concordant with previous studies. ${ }^{4,7} 8$ On the other hand, the $14 \%$ prevalence of psychogenic pseudo-syncope in our study is at variance with other studies, which reported $<6 \%$ psychogenic origins of syncope. ${ }^{2,6}$ Others, however, have underestimated its true prevalence, which might be as high as $24 \%$ in a population presenting with unexplained syncope., Patients with psychogenic pseudo-syncope were rather young, women, and frequently suffered from presyncope, suggesting that psychiatric manifestations should be routinely sought in patients with unexplained syncope, and that psychogenic pseudo-syncope is not only a diagnosis of exclusion but can be firmly established. Following the standardized diagnostic evaluation, $1 / 3$ of our patients remained without an explained cause. They shared some common characteristics with arrhythmic causes, such as a high prevalence of abrupt onset $(43 \%)$ compared with other categories. In 
54 of these patients an IRL was implanted and, surprisingly, multiple etiologies were finally established.

\section{Diagnostic Yield of a Standardized Stepwise Evaluation}

The diagnostic approach of patients with unexplained syncope is a clinical challenge. Despite multiple investigations, syncope remains unexplained in up to approximately $50 \%$ of cases. $^{1,2}$ The superiority of standardized investigation strategies of unexplained syncope over usual practice has been recently shown in patients with syncope admitted to emergency departments. ${ }^{1-3}$ The present study suggests that a standardized stepwise diagnostic evaluation establishes an etiology in $2 / 3$ of patients referred for unexplained syncope to an outpatient clinic, a proportion concordant with other studies, ${ }^{2,4}$ and highlights the use of noninvasive tests for the investigation of unexplained recurrent syncope. In selected patients, however, ILR established a diagnosis in over $50 \%$ of recipients, confirming its incremental benefit in patients with unexplained syncope after a negative standardized diagnostic evaluation.

\section{Clinical Characteristics and Distribution of Prodromes According to Causes of Syncope}

Alboni et al. and Sheldon et al. recently observed an older age and fewer prodromes in patients with carotid sinus hypersensitivity or cardiac syncope compared with patients who present with other forms of neurally mediated syncope., ${ }^{7,8}$ Our study extends these observations to other causes of cardiac syncope. Patients with arrhythmic events were older, had a higher prevalence of hypertension and ischemic heart disease, and re-

\section{References}

1. Sarasin FP, Louis-Simonet M, Carballo D, Slama S, Rajeswaran A, Metzger JT, Lovis C, et al. Prospective evaluation of patients with syncope: A population-based study. Am J Med 2001; 111:177184.

2. Farwell DJ, Sulke AN. Does the use of a syncope diagnostic protocol improve the investigation and management of syncope? Heart 2004; 90:52-58.

3. Brignole M, Disertori M, Menozzi C, Raviele A, Alboni P, Pitzalis MV, Delise P, et al. Management of syncope referred urgently to general hospitals with and without syncope units. Europace 2003; 5:293-298.

4. Graf D, Schlaepfer J, Gollut E, van Melle G, Mischler C, Fromer M, Kappenberger L, et al. Predictive models of syncope causes in an outpatient clinic. Int J Cardiol 2008; 123:249-256.

5. Brignole M, Alboni P, Benditt DG, Bergfeldt L, Blanc JJ, Bloch Thomsen PE, van Dijk JG, et al., on behalf of the Task Force on Syncope, European Society of Cardiology). Guidelines on management (diagnosis and treatment) of syncope: Update 2004. Europace 2004; 6:467-537. ported fewer prodromes. Their functional capacity was lower and $\mathrm{P}$-wave duration longer than in patients with reflex syncope. This study of $>900$ patients confirms our previous observations ${ }^{4}$ that clinical characteristics, syncope presentation, and P-wave duration may be used to identify a subgroup of patients more likely to suffer from arrhythmic syncope.

\section{Limitations of the Study}

The first limitation of our study was the absence of objective criteria for the diagnosis of psychogenic pseudo-syncope, which may have overestimated the true prevalence of this disorder. Final diagnoses, however, were established when the test-induced symptoms strictly matched the clinical event. Second, Krahn et al. recently reported a higher cost-effectiveness of the ILR over conventional testing in a similar design. ${ }^{11}$ The diagnostic yield of conventional testing was surprisingly low $(20 \%)$ compared with the present study (66\%). The effectiveness of our stepwise evaluation needs to be addressed and compared with other investigation strategies in terms of recurrence and cost.

\section{Conclusions}

A standardized stepwise diagnostic evaluation focusing on noninvasive tests identified two-thirds of causes in patients referred to an ambulatory clinic for unexplained syncope. Vasovagal syncope and psychogenic pseudo-syncope accounted for $>50 \%$ of all causes, while cardiac arrhythmias were uncommon. Sudden syncope, especially in older patients with functional limitations or a prolonged P-wave duration, suggested an arrhythmic cause.

6. Brignole M, Menozzi C, Bartoletti A, Giada F, Lagi A, Ungar A, Ponassi I, et al. A new management of syncope: Prospective systematic guideline-based evaluation of patients referred urgently to general hospitals. Eur Heart J 2006; 27:76-82.

7. Alboni P, Brignole M, Menozzi C, Raviele A, Del Rosso A, Dinelli $\mathrm{M}$, Bettiol K, et al. Clinical spectrum of neurally mediated reflex syncopes. Europace 2004; 6:55-62.

8. Sheldon R, Rose S, Connolly S, Ritchie D, Koshman ML, Frenneaux M. Diagnostic criteria for vasovagal syncope based on a quantitative history. Eur Heart J 2006; 27:344-350.

9. Linzer M, Felder A, Hackel A, Perry AJ, Varia I, Melville ML, Krishnan KR. Psychiatric syncope: A new look at an old disease. Psychosomatics 1990; 31:181-188.

10. Benbadis SR, Chichkova R. Psychogenic pseudosyncope: An underestimated and provable diagnosis. Epilepsy Behav 2006; 9:106110.

11. Krahn AD, Klein GJ, Yee R, Hoch JS, Skanes AC. Cost implications of testing strategy in patients with syncope: A randomized assessment of syncope trial. J Am Coll Cardiol 2003; 42:495-501. 